\title{
The use of renin-angiotensin-aldosterone system inhibitors in chronic kidney disease: Is there any doubt?
}

\author{
Ana Carina Ferreira1,2 \\ ${ }^{1}$ Nephrology Department, Centro Hospitalar e Universitário de Lisboa Central, Hospital Curry Cabral, Lisbon, Portugal \\ ${ }^{2}$ Nova Medical School, Nova University, Lisbon, Portugal
}

Renin-Angiotensin-Aldosterone system (RAAS) is of utmost importance in volume and blood pressure control, as well in sodium homeostasis $^{1}$. The classical event for RAAS activation is volume depletion. Its presence activates arterial baroreceptors, namely the ones present in the renal afferent arterioles that will lead to renin secretion by the juxtaglomerular apparatus. Renin transforms angiotensinogen in angiotensin I, which is converted in angiotensin II (AngII) by angiotensin-converting enzyme (ACE). In its turn, Angll causes vasoconstriction of renal efferent arteriole, as well as increases in sodium resorption by proximal tubule, and indirectly causes antidiuretic hormone release. In the suprarenal cortex, Angll induces the aldosterone synthesis, which acts in the principal cells of collector ducts, causing resorption of sodium and excretion of potassium (through the increased expression of $\mathrm{ENaC}$ and ROMK), and increased secretion of prostaglandins ${ }^{1}$.

Despite its importance, the chronic stimulation of RAAS is deleterious, promoting endothelial dysfunction, inflammation and fibrosis ${ }^{2}$, and physicians are aware that its blockage is very efficient in controlling high blood pressure.

Nephrologists are also aware of the importance of RAAS inhibition (RAASi) to the preservation of glomerulus health. If we block the vasoconstriction of renal efferent arteriole promoted by Angll, we will diminish the intra-glomerular pressure, and this protects kidneys in the long term. Nevertheless, this system inhibition is not free of complications. The drop in intra-glomerular pressure can precipitate a reduction in the glomerular filtration rate (GFR), which is more likely to happen in a subgroup of patients, namely the ones with chronic kidney disease (CKD) or with heart failure, who are the ones who probably most benefit from RAASi. In addition, RAASi can cause hyperkalemia, and this life-threatening event is more frequent in the presence of CKD.

\section{THE USE OF RAASI IN ADVANCED CKD}

Despite possible side effects, the use of RAASi in CKD has been recommended for several years. In 2012 the Kidney Disease | Improving Global Outcomes (KDIGO) guidelines suggested the use of ACEi or ARB in both diabetic and non-diabetic patients with CKD and a urine albumin excretion above $300 \mathrm{mg} / 24 \mathrm{~h}$, with a $1 B$ level for the recommendation ${ }^{3}$.

More recently, in 2020, the KDIGO guidelines for Diabetes in CKD advocated treatment with ACEi or ARB in albuminuric hypertensive patients, with an evidence level of $1 B^{4}$. The use of those drugs could be considered in the absence of high blood pressure and there seems to be no advantage in its use in the absence of albuminuria ${ }^{4}$.

This year has seen the launch of the KDIGO guidelines for blood pressure in non-dialysis $C K D^{5}$. The recommendations for patients with high blood pressure and CKD were different, depending on the presence of diabetes: in diabetic patients, it was recommended to use RAASi in the presence of albuminuria (level of evidence 1B); in non-diabetic patients it was recommended to use RAASi with severely increased albuminuria (A3 - level of evidence $1 B$ ) or with moderately increased albuminuria ( $\mathrm{A} 2$ - level of evidence $2 \mathrm{C})^{5}$.

The prevention of CKD progression is the goal of all nephrologists. For almost 20 years, we have known the value of RAASi in achieving this goal. The IDNT ${ }^{6}$ and the RENAAL ${ }^{7}$ landmark studies were published in 2001 and both showed that, in a population with type 2 diabetes, irbesartan and losartan, respectively, were important in preventing death, end-stage renal disease (ESRD) or duplication of creatinine, with a risk reduction of $20 \%$ and $16 \%$, correspondingly. Moreover, the AASK study ${ }^{8}$, published in 2002, showed the importance of ramipril in the progression of CKD beyond its antihypertensive effects, in African Americans. A further 3 landmark studies, HOPE ${ }^{9}$, EUROPA ${ }^{10}$, and PEACE ${ }^{11}$, showed the importance of ACE inhibitors (ACEi) and angiotensin II receptor blockers (ARB) in cardiovascular protection in patients with and without hypertension, including CKD patients. Nevertheless, those benefits are dependent on the dose we use, and it is very difficult to use full-dose ACEi or ARB in CKD patients.

With the above in mind, are there any studies in the subgroup of patients with advanced kidney disease? Yes, three observational studies have just been published. And I immediately acknowledge a common limitation in these three studies: as they are observational, we cannot infer causality.

The first study I chose to talk about is a nationwide Swedish observational study ${ }^{12}$, which included a cohort of patients with an estimated GFR (eGFR) below $30 \mathrm{ml} / \mathrm{min} / 1.73 \mathrm{~m}^{2}$. The authors compared the introduction of RAASi versus calcium channel blockers and follow 4803 patients for 4.1 years. They verified that those patients with advanced kidney disease benefitted from the introduction of RAASi, as it slowed CKD progression, with no differences in mortality or cardiovascular events.

The same authors published another study based on the same Swedish Renal Registry, including 10,254 patients with an eGFR below 
$30 \mathrm{ml} / \mathrm{min} / 1.73 \mathrm{~m}^{2}$, this time under an $\mathrm{ACEi}^{13}$. The aim was to compare patients who stopped the medication within 6 months versus patients who continued the medication, through five years of follow-up. Stopping RAASi was associated with a higher risk of mortality and major cardiovascular events, but associated with a lower absolute risk of starting dialysis.

A third analysis performed in the USA studied the long-term effects of RAASi discontinuation in non-dialysis CKD. The authors included 141,252 patients who were prescribed with ACEi or ARB for the first time $^{14}$. From these, 135,346 had a drug discontinuation event, although $61 \%$ restarted the drug within 6 months. The authors concluded that discontinuation of the drugs over any duration (14-30 days or $>180$ days) was associated with an increase hazard ratio of death and ESRD.

It seems that the benefits of these drugs in CKD patients outweighs the risks of lowering their eGFR.

We should not forget about the importance of the aldosterone blockage, in an attempt to reduce the aldosterone escape, which can occur in 10 to $40 \%$ of people using ACEi or ARB ${ }^{15}$. A new nonsteroidal mineralocorticoid receptor antagonist was developed: finerenone. This drug causes less hyperkalemia than spironolactone ${ }^{16}$. Two studies were developed: one focused on the reduction of renal events (FIDELIO-DKD), and the other focused on cardiovascular morbidity and mortality (FIGARO-DKD), both in type 2 diabetes. The first one included 5,674 diabetic patients with a baseline eGFR of $44 \mathrm{ml} /$ $\mathrm{min} / 1.73 \mathrm{~m}^{2}$, and a median urinary albumin-creatinine ratio of $852 \mathrm{mg} / \mathrm{g}$. It showed that, on top of standard care (which includes an ACEi or ARB), aldosterone blockage with finerenone reduced by $18 \%$ the risk ratio of sustained decline in eGFR ( $\geq 40 \%)$, dialysis initiation, or renal death ${ }^{17}$, with no superior risk of acute kidney injury compared to placebo. The results of the second one are not divulgated yet. Additionally, aldosterone blockage is being studied in ESRD, in which there are 2 randomized control studies on-going: the ALCHEMIST, and the ACHIEVE, that will focus on cardiovascular events in dialysis patients ${ }^{18}$.

\section{HYPERKALEMIA WITH THE USE OF RAASI}

Currently, the management of RAASi in CKD patients is easier since we have new potassium binders with benefits when compared to the classical resins.

Patiromer and sodium zirconium cyclosilicate are game changers in the use of RAASi in CKD patients. Patiromer is a non-absorbed drug, which acts mostly in the distal colon through a non-specific cationic-exchange (calcium-potassium). Its action starts within 7 hours, and patients achieve normokalemia mostly in 4 weeks. It is well-tolerated, can cause hypomagnesemia in some patients and must be taken 3 hours apart from the rest of the medication ${ }^{19}$.

Zirconium cyclosilicate exchanges potassium for hydrogen and sodium through the entire gastrointestinal tract. Most patients achieve normokalemia in $24 \mathrm{~h}$. It is a well-tolerated drug, although it can cause edema. As with patiromer, it must be taken apart from the rest of the medication, usually with an interval of 2 hours ${ }^{20}$.

With these recent publications and new potassium binders, there is no excuse for letting the old drugs (and the new ones) act for the benefit of CKD patients by blocking RAAS.

\section{References}

1. Gonwa TA, Wadei HM. Kidney disease in the setting of liver failure: core curriculum 2013. Am J Kidney Dis. 2013;62(6):1198-1212.

2. Buonafine M, Bonnard B, Jaisser F. Mineralocorticoid receptor and cardiovascular disease. Am J Hypertens. 2018;31(11):1165-1174.

3. KDIGO 2012 clinical practice guideline for the evaluation and management of chronic kidney disease. Kidney Int. 2013(3):1-150.

4. KDIGO 2020 Clinical Practice Guideline for Diabetes Management in Chronic Kidney Disease. Kidney Int. 2020;98(4s):s1-s115.

5. KDIGO 2021 Clinical Practice Guideline for the Management of Blood Pressure in Chronic Kidney Disease. Kidney Int. 2021;99(3s):s1-s87.

6. Lewis EJ, Hunsicker LG, Clarke WR, et al. Renoprotective effect of the angiotensin-receptor antagonist irbesartan in patients with nephropathy due to rype 2 diabetes. $N$ Engl J Med. 2001;345(12):851-860.

7. Brenner BM, Cooper ME, de Zeeuw D, et al. Effects of losartan on renal and cardiovascular outcomes in patients with type 2 diabetes and nephropathy. N Engl J Med. 2001;345(12):861-869.

8. Wright JT, Jr., Bakris G, Greene T, et al. Effect of blood pressure lowering and antihypertensive drug class on progression of hypertensive kidney disease: results from the AASK trial. JAMA. 2002;288(19):2421-2431.

9. Yusuf S, Sleight P, Pogue J, Bosch J, Davies R, Dagenais G. Effects of an angiotensin-converting-enzyme inhibitor, ramipril, on cardiovascular events in high-risk patients. $N$ Engl J Med. 2000;342(3):145-153.

10. Fox KM. Efficacy of perindopril in reduction of cardiovascular events among patients with stable coronary artery disease: randomised, double-blind, placebo-controlled, multicentre trial (the EUROPA study). Lancet. 2003;362(9386):782-788

11. Angiotensin-Converting-Enzyme Inhibition in Stable Coronary Artery Disease. New England Journal of Medicine. 2004;351(20):2058-2068.

12. Fu EL, Clase CM, Evans M, et al. Comparative effectiveness of renin-angiotensin system inhibitors and calcium channel blockers in individuals with advanced CKD: a nationwide observational cohort study. Am J Kidney Dis. 2021;77(5):719-729.e711.

13. Fu EL, Evans M, Clase CM, et al. Stopping renin-angiotensin system inhibitors in patients with advanced CKD and risk of adverse outcomes: a nationwide study. J Am Soc Nephrol. 2021;32(2):424-435

14. Walther CP, Winkelmayer WC, Richardson PA, Virani SS, Navaneethan SD. Renin-angiotensin system blocker discontinuation and adverse outcomes in chronic kidney disease. Nephrol Dial Transplant. 2020.

15. Bomback AS, Klemmer PJ. The incidence and implications of aldosterone breakthrough. Nat Clin Pract Nephrol. 2007;3(9):486-492.

16. Rico-Mesa JS, White A, Ahmadian-Tehrani A, Anderson AS. Mineralocorticoid receptor antagonists: a comprehensive review of finerenone. Curr Cardiol Rep. 2020;22(11):140.

17. Bakris GL, Agarwal R, Anker SD, et al. Effect of finerenone on chronic kidney disease outcomes in type 2 diabetes. N Engl J Med. 2020;383(23):2219-2229.

18. Rossignol P, Frimat L, Zannad F. The safety of mineralocorticoid antagonists in maintenance hemodialysis patients: two steps forward. Kidney Int. 2019;95(4):747-749.

19. Colbert GB, Patel D, Lerma EV. Patiromer for the treatment of hyperkalemia. Expert Rev Clin Pharmacol. 2020;13(6):563-570.

20. Takkar C, Nassar T, Qunibi W. An evaluation of sodium zirconium cyclosilicate as a treatment option for hyperkalemia. Expert Opin Pharmacother. 2021;22(1):19-28.

\section{ORCID}

Ana Carina Ferreira (iD) 0000-0002-1323-5293

\section{Correspondence to:}

\section{Ana Carina Ferreira, MD}

Nephrology Department, Centro Hospitalar e Universitário de Lisboa Central Hospital Curry Cabral, Lisbon, Portugal

Rua da Beneficência no 8; 1050-099 Lisbon, Portugal

E-mail: carina.ferreira@fcm.unl.pt 